\title{
The relationship between head and eye movement in congenital nystagmus with head shaking: objective recordings of a single case
}

\author{
M. GRESTY, G. M. HALMAGYI, AND J. LEECH \\ From the Medical Research Council Hearing and Balance Unit, Institute of Neurology, and Department \\ of Neuro-ophthalmology, National Hospital, London
}

SUMMARY Head shaking and congenital nystagmus were recorded in a patient presented with visual tasks. When she was at rest the nystagmus took a 6 cycles per second saw-tooth wave-form. When she was attentive the nystagmus beat at a 2 to 2.6 cycles per second with a saddle-shaped deformation which permitted foveation. The head shaking occurred occasionally when the patient was attentive and was phase-locked to the nystagmus with resemblances in wave form and direction. Deceleration of the head shaking to zero velocity and peak displacement (to the left) coincided with the onset of the saddle of the nystagmus and hence assisted foveation; all other parts of the head-shaking cycle were detrimental to vision. It is proposed that the head shaking has a common pathological origin with the nystagmus and that, just as an isolated congenital nystagmus wave form becomes altered with attention to permit periods of foveal fixation, the pattern of combined head and eye nodding in this patient provided similar periods of fixation.

The specific association of head nodding with congenital nystagmus was first described by Hadden (1890) and Aldrich (1899), and implicit in the discussion of their cases is the notion that the origin of the head nodding was pathological, not compensatory. Since the early descriptions many attempts the have been made to elucidate the nature of the nystagmus. The head nodding, however, has received little attention. The reason for the neglect is twofold. Firstly, only recently have objective measurements of movement co-ordination been available in clinical neurology, and secondly, as Hadden observed, the occurrence of nodding with congenital nystagmus is very variable, being subject to states of arousal and anxiety and attention. Hence it is difficult to provoke a patient with congenital nystagmus and nodding to perform at will.

Prompted by Professor Louis Dell'Osso, we searched among our patients for someone whose head nodding appeared frequently, and having found such a subject we made objective recordings of her head and eye movements to investigate the shape of the nodding wave form and its relationship to the nystagmus.

Address for reprints: Dr G. M. Halmagyi, MRC Hearing and Balance Unit, Institute of Neurology, National Hospital, Queen Square, London WC1N 3BG

\section{Methods and material}

The technique employed to transduce head and eye movements has been described by Gresty et al (1976). Briefly, the patient sat in a high-backed armchair which restricted torso movement, facing a lectern which was used to support various types of visual display. She was tested with her spectacles in ambient laboratory illumination. Eye movements were recorded using DC electro-oculography and calibrated by stepping the eyes through fixed target displacements. Head movements were recorded by fixing to the occiput a small lamp which was viewed by a dual axis Schottky barrier photodetector (United Detector technology SC/50) mounted with its sensitive surface in the focal plane of a $35 \mathrm{~mm}$ camera. The head was calibrated by being stepped through predetermined angular displacements.

The patient was presented with the task of reading newsprint placed on the lectern and of reading single numerals of a light-emitting diode display which generated random numbers at the push of a button held in the patient's hand. The light-emitting diode numeral subtended visual angles of arc 0.006 vertically and arc 0.003 horizontally. The patient was examined during two 2-hour sessions 1 week apart. Her nodding was much reduced on the 
second session and she reported that she was much more at ease.

\section{CASE REPORT}

The patient, a woman aged 33, presented in August 1977 to the National Hospital, Queen Square, London, for neuro-ophthalmological assessment regarding the possibility of improving her vision. She was a high myope with corrected distance vision $6 / 24$, N 4.5 near vision in each eye, and normal colour vision. Nystagmus was noted in early life and had persisted ever since. The associated head nod was quite prominent in childhood and adolescence but had largely disappeared in adult life apart from times of concentration, especially during attempted fixation, for example, reading. There was no family history of nervous or eye disease.

There was a high amplitude horizontal nystagmus which was suppressed in extreme down gaze but not by convergence. No ocular transillumination was observed. The retina had the appearances of myopia but was not degenerative or albinotic. Occasional irregular movement of head were noted during the early parts of interview and examination. No other neurological or medical abnormality was detected.

\section{EXPERIMENTAL FINDINGS}

During all recordings both head nodding and nystagmus were confined to the horizontal plane (yaw). For the sake of brevity only the essential findings are listed.

\section{Shape of congenital nystagmus wave forms}

(a) When at rest the patient had a saw-toothshaped nystagmus wave form, of which the fast phases beat predominantly to the right. The mean frequency of beating was 6 per second with little variation (Fig. 1).

(b) When the patient concentrated on reading the light-emitting diode display, the nystagmus transformed to the so-called saddle shape (the "jerk nystagmus with extended foveation' of Dell'Osso and Daroff, 1975) (Fig. 2; Fig. 3, reading lightemitting diode). Fast phases beat to the left at a frequency varying between 2.5 and 2 per second. In between fast phases, when the eyes were towards the left, a saddle-shaped movement occurred (Fig. 3, reading light-emitting diode) giving the impression of the presence of a second harmonic distortion at a frequency of approximately 5 cycles per second.

\section{Shape of the head movement wave form}

The head shaking was of small amplitude, pendular, and asymmetrical, with the faster movements executed to the right (Fig. 2; Fig. 3, reading lightemitting diode). At times there was a distortion on

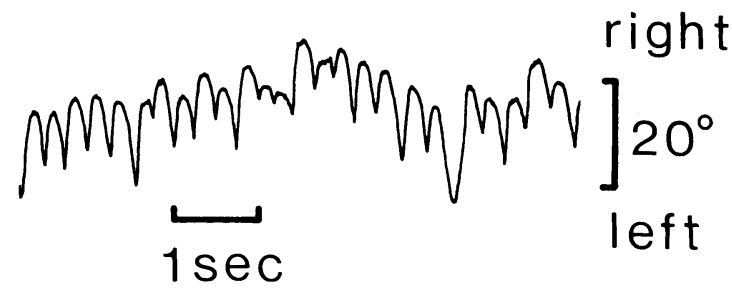

Fig. 1 Eye movement recordings from a patient with congenital nystagmus and head nodding showing the shape of the congenital nystagmus wave form when the patient was at rest with no immediate visual task. Head movement was absent

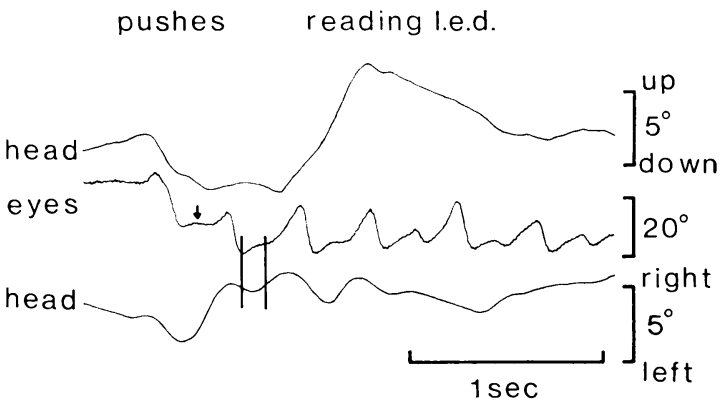

Fig. 2 Freely moving head and eye movement recordings from a patient with congenital nystagmus and head nodding. The patient sat in front of a (single lightemitting diode (l.e.d.)) display consisting of a numeral. When the patient pushed a button held in her hand the display generated a random number which the patient read aloud. Arrow indicates the saddle of the nystagmus wave form; parallel lines indicate the zone in which foveal fixation probably occurs

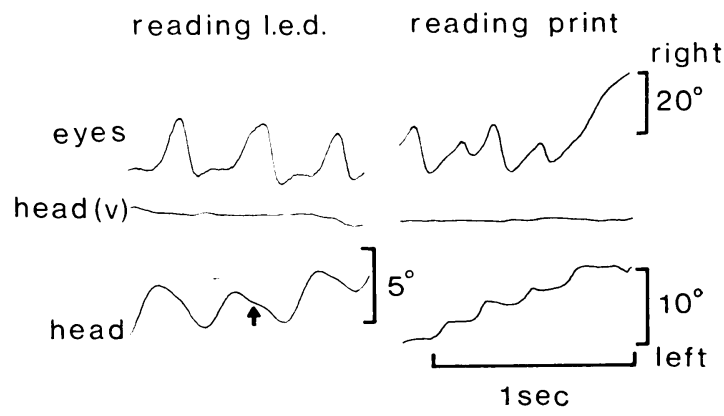

Fig. 3 Experimental situation as in Fig. 1 together with recordings from freely moving head and eyes while the patient read newsprint. Arrow indicates higher frequency distortion of head movement wave form 
the head movement wave form which occurred during the leftwards movement and resembled the saddle of the congenital nystagmus wave form. The beating frequency of the head shaking varied between 2 and 2.6 cycles per second. The head shaking appeared to be more or less phase-locked to the nystagmus, such that peak eye displacement to the left occurred approximately $50 \mathrm{~ms}$ before peak head displacement to the left.

\section{Print reading}

When the patient read newsprint, she used stepping movements of the head to scan the print rather than stepping eye movements (Fig. 3, reading print).

\section{Discussion}

Dell'Osso and Daroff (1975) showed that target foveation is achieved during a particular part of the congenital nystagmus wave form, which in the case of the nystagmus presented by our patient is the flat part of the saddle during which the eyes were stationary for the longest time. Just before the saddle the eyes moved with low velocity towards the right, at which time the head was moving at a similar velocity leftwards towards peak displacement. Thus during and just preceding the saddle the head and eyes were in more or less compensatory relationship, providing a period of stable vision. During all other parts of the cycle the head and eyes moved either in the same direction or with varying relationships, neither of which could provide stable vision. We are left with a situation in which part of the head movement is compensatory for the nystagmus and most of it detrimental to visual acuity.

There are 2 possible explanations for the patterns of head and eye co-ordination observed in this patient. In the first place the head nodding may occur purely as an attempt to compensate for the nystagmus and, because of the complexity of the nystagmus wave form, provides compensation only at certain intervals. The negative evidence for this explanation is that the nodding wave form is itself irregular and contains components which are inappropriate, and that the onset of nodding is closely related to situations of stress rather than any requirement for fine visual discrimination.

Alternatively it may be assumed that the nodding, like the nystagmus, is pathological in origin, in which case, just as the wave form of an isolated congenital nystagmus becomes modified during visual attention to provide periods of visual stability, the combination of nodding and nystagmus in the present patient provides similar periods which permit stable foveation. It may be that modification of the nystagmus occurs through the action of the doll's head reflex compensating for the nodding.

As to the nature of the mechanism which makes extended periods of foveation possible, it may well be a cancellation of movement due to beating between the principal frequency component(s) of the nystagmus wave form and the higher frequency harmonic(s).

\section{References}

Aldrich, C. J. (1899). Head nodding and head rotation usually associated with nystagmus in very young children. American Journal of the Medical Sciences, 117, $158-168$.

Dell'Osso, L. F., and Daroff, M. D. (1975). Congenital nystagmus wave forms and foveation strategy. Documenta Ophthalmologica, 39, 155-182.

Gresty, M., Leech, J., Sanders, M., and Eggars, H. (1976). A study of head and eye movement in spasmus nutans. British Journal of Ophthalmology, 60, 652-654.

Hadden, W. B. (1890). On head nodding and head jerking in children, commonly associated with nystagmus. Head jerking in children, a second series of cases. Lancet, 1 , 1293,1349 , and 1416 , reprinted from Vol. XX of the St. Thomas's Hospital Reports. 